\title{
Simulation Based Elevator Group Control System for Multi-Storey Building
}

\author{
Bharat Bhushan Sharma \\ Department of Electronics \& Instrumentation, School of Automation \\ Banasthali Vidyapith, Rajasthan, India \\ Anuj Banshwar*, Mohit Pathak \\ Department of Electrical \& Electronics Engineering \\ Noida Institute of Engineering \& Technology, Greater Noida, India \\ *Corresponding author: anujbanshwar@gmail.com \\ Naveen Kumar Sharma \\ Department of Electrical Engineering \\ I. K. Gujral Punjab Technical University, Jalandhar, Punjab, India \\ Aman Joshi \\ Department of Physics \\ National Institute of Technology Kurukshetra, Kurukshetra, India \\ (Received April 29, 2018; Accepted November 18, 2018)
}

\begin{abstract}
In this work fuzzy logic based controller for Elevator Group Control System (EGCS) is proposed. The most complex problem is to control the multi-elevator in a multi-storey building and there are different approaches to solve the problem. The approach used based on the Simulink model and the fuzzy logic controller which is proposed to choose the most appropriate elevator for a hall call generated by passenger/user. The simulation was carried out by giving the input which was used to calculate the input for fuzzy logic controller. The data obtained from the simulation model and the input source provided to the fuzzy based controller priority of each elevator car can be calculated. The maximum priority elevator car is responded for its hall call operation. The input was based on hall call assignment method. This work can assist in analyzing the performance of the Elevators.
\end{abstract}

Keywords- Fuzzy logic controller, Simulink, Hall call assignment method, Elevator group control system.

\section{Introduction}

An EGCS is used for automation of three or more elevators in a group. An EGCS is useful in reducing the waiting time and riding time and to provide an increased quality of service to passengers. The EGCS consists of various parameter calculations such as waiting time, riding time, travelling distance, hall call area weight, destination area weight. This parameter acts as the input to the Fuzzy Logic Controller (FLC) (Zadeh, 1973; Lee, 1990). In general, EGCS uses the hall call assignment method (HCAM) which designates elevators in response to a passenger's call. In this case, the EGCS selects the nearly available elevator to the request of the passenger. The HCAM assigns a new hall call to the elevator when the user/passenger gives their destination details before entering in the elevator car. This method was very helpful as it reduces the ambiguity of the destination and reduces the fake calls which were assigned by the passengers after entering the elevator car. The area-weight is an important parameter and affects the evaluation function values of elevators. In this work, the simulation method is implemented. In 
International Journal of Mathematical, Engineering and Management Sciences

Vol. 4, No. 1, 77-84, 2019

https://dx.doi.org/10.33889/IJMEMS.2019.4.1-007

general, it is very complex to create an exact replica to manage a system with higher complexity and in this type of system accurate calculation of system state is complicated (Nikovski and Brand, 2004; Patjoshi and Mahapatra, 2010). For this type of system, approximation methods on fuzzy system can be implemented (Chaube and Singh, 2016a; 2016b). It has been reported that many application of fuzzy based advanced controllers were implemented in elevator control. In this work, system space and expert knowledge are described by the fuzzy model and area weight parameter is determined by the fuzzy inference method (Imasaki et al., 1995; Ishikawa et al., 2000). It is seen that fuzzy system has shown enhanced outcome than the predictable scheme.

\section{Hall Call Assignment Method Based Elevator System}

In this elevator system, a twenty-digit keypad is installed in the hall of each floor where the passengers enter their destination floor before entering in the elevator car. When the destination was identified priory and no extra call can be assigned by the traveller while travelling within the elevator (Kim et al., 2000; King and Bouketir, 2008). This will provide almost entire and precise data of the current traffic situation and it will make the work of EGCS easy in dispatching the elevator for particular assigned information. After receiving destination, the EGCS selects the elevator having the maximum priority i.e. best suited elevator for the destination by FLC. A display informs passenger which elevator was assigned for his/her destination. In this system, an elevator group control was designed for destination-oriented elevator system as illustrated in Figure 1.

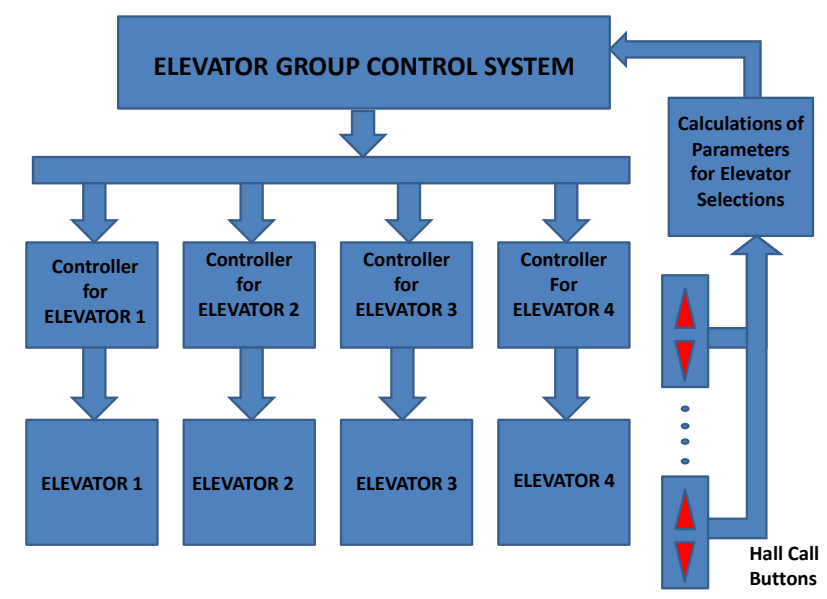

Figure 1. Block diagram of EGCS

EGCS is an uncertain non-linear complex decision making problem with randomness. Subsequently, EGCS has to be multi-purpose to achieve the preferred performance. The methodology used in EGCS is nearly fuzzy because it consists of several inputs such as traveller waiting time, number of the traveller in the elevator, the response time for calling. These inputs are not précised and cannot be defined accurately. Therefore it is very difficult to deal with these problems with the basic logic sets and in this work the fuzzy based rules are designed to solve this complex problem. Thus, EGCS can be modulated with fuzzy inference. In general, the fuzzy 
International Journal of Mathematical, Engineering and Management Sciences

Vol. 4, No. 1, 77-84, 2019

https://dx.doi.org/10.33889/IJMEMS.2019.4.1-007

inference is based on the experience gained by the experts. In EGCS based on fuzzy control all the traffic flow such as peak hour, normal traffic, idle flow, number of traveller, traveller waiting time and consumption of energy are considered (Kim, 2000; Deying and Dongmei, 2010). In lieu of providing the optimal solution for the allocation of elevators, an optimized function is designed. The methodology consists of four essential units. First is input which is elevator call, second is calculation unit for various parameters. The third unit is fuzzy based control called as a fuzzy control unit and last is the most important selection or best solution sorting unit.

\section{A. Input to the Elevator System}

The elevator system is given the input in the form of hall call by the hall call assignment method in numerical form.

\section{B. Parameter calculation}

The five parameters are a time of wait, time of ride, distance travelled, the weight of hall call area, weights of the destination area. These parameters are calculated by the parameter calculation block and these parameters are calculated for each elevator. These parameters act as the input for the fuzzy logic controller.

\section{Fuzzy Control Unit}

- Fuzzy control: Sugeno (1985) demonstrated the very first application of fuzzy control in the laboratory. Kickert and Mamdani (1993) worked out the first fuzzy control technology. In a controlling system for cement kiln, the first large-scale realization of a fuzzy controller was used. In early 1990, various thriving engineering applications were reported from Japan in the field of customer goods like automatic washing machines, cranes, elevators, vacuum cleaners and various power plants (Tanaka and Watanabe, 2009).

It is an important intelligent control and has various features. It is suitable for non-linear control system because it has a non-linear control technique. It is fit for multifaceted models that require a higher degree of accuracy in terms of energy utilization. It utilizes the experience of engineering expert and provides a suitable design using an FLC. In FLC, it is easier to select the parameters and the analytical control is speedy, responsive and simple.

- Fuzzy Inference systems: In MATLAB there is built in Fuzzy inference systems which can be utilized for the various parameters. In this parameter calculation block, one can get the desired output with provided inputs. Input can be whose input is the output of the parameter calculation block. The inputs are time of wait, time of ride, distance travelled, the weight of hall call area, weights of the destination area. Theses inputs of the FLC system are ranged as and can be defined as:
Time of wait from one to thirty;
$>$ Time of ride from one to thirty;
$>$ Distance travelled from one to twenty;
Weight of hall call area from one to twenty;
$>$ Weights of destination area from one to five.

The priority for the FLC system and their range of output are from zero to one. 
International Journal of Mathematical, Engineering and Management Sciences

Vol. 4, No. 1, 77-84, 2019

https://dx.doi.org/10.33889/IJMEMS.2019.4.1-007

BLOCK DIAGRAM OF ELEVATOR SYSTEM

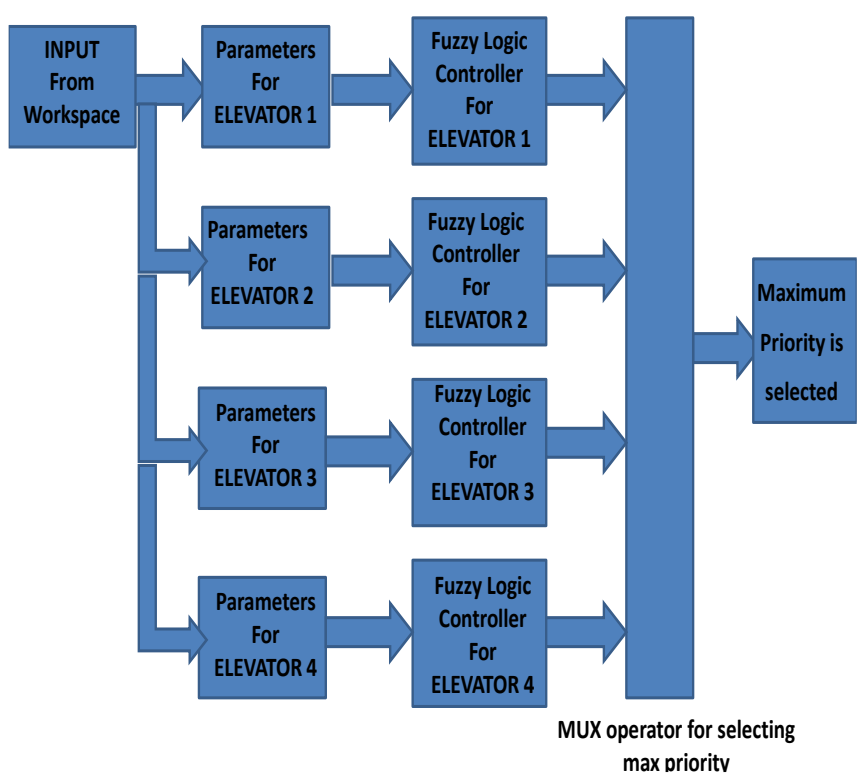

Figure 2. Block diagram of the simulation technique

Figure 2 depicts the block diagram of the simulation technique used in this work. Figure 3 shows the fuzzy system used in the work with various inputs and priority as output. Each input is provided with a membership function with predefined values and Sugeno based FLC named elevatanfs is shown in Figure 3 and inference rules of the fuzzy system with their weights are given in Table 1.

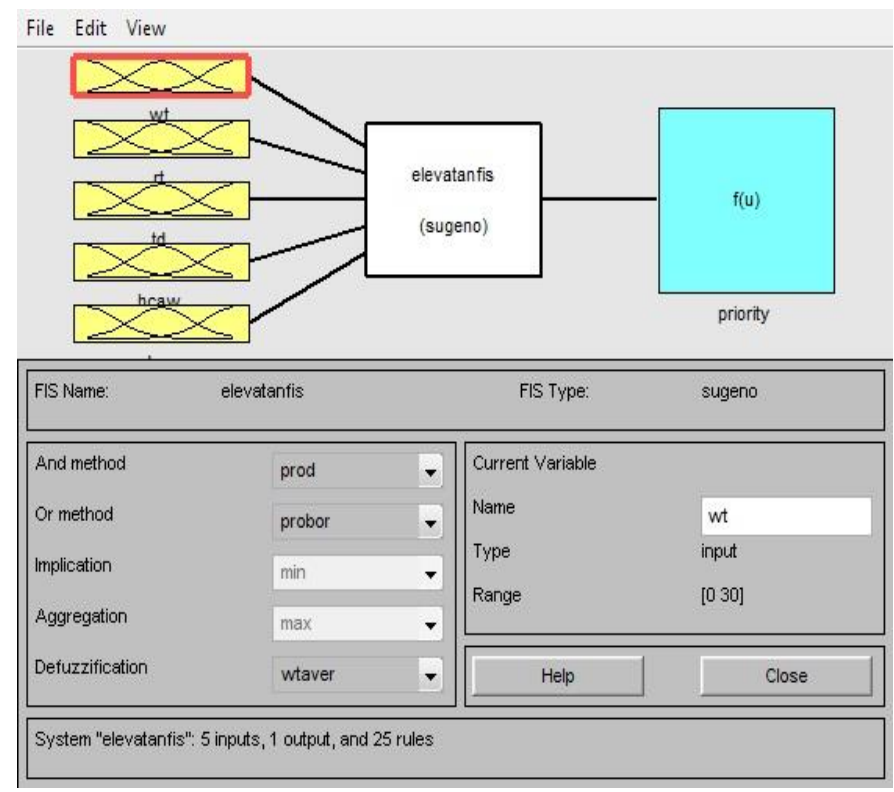

Figure 3. Fuzzy system for EGCS 
International Journal of Mathematical, Engineering and Management Sciences

Vol. 4, No. 1, 77-84, 2019

https://dx.doi.org/10.33889/IJMEMS.2019.4.1-007

Table 1. Inference rules of the fuzzy system with their weights

\begin{tabular}{|l|c|}
\hline \multicolumn{1}{|c|}{ Inference Rules } & Weights \\
\hline If the waiting time is Vshort then priority is Vbig & 0.7 \\
\hline If the waiting time is short then priority is big & 0.7 \\
\hline If the waiting time is med then priority is med & 0.7 \\
\hline If the waiting time is big then priority is small & 0.7 \\
\hline If the waiting time is Vbig then priority is Vsmall & 0.7 \\
\hline If riding time is Vshort then priority is Vbig & 0.7 \\
\hline If riding time is short then priority is big & 0.7 \\
\hline If riding time is med then priority is med & 0.7 \\
\hline If riding time is big then priority is small & 0.7 \\
\hline If riding time is Vbig then priority is Vsmall & 0.7 \\
\hline If the travelling distance is Vshort then priority is Vbig & 0.05 \\
\hline If the travelling distance is short then priority is big & 0.05 \\
\hline If the travelling distance is med then priority is med & 0.05 \\
\hline If the travelling distance is big then priority is small & 0.05 \\
\hline If the travelling distance is Vbig then priority is Vsmall & 0.05 \\
\hline If hcaw is Vshort then priority is Vbig & 0.6 \\
\hline If hcaw is short then priority is big & 0.6 \\
\hline If hcaw is med then priority is med & 0.6 \\
\hline If hcaw is big then priority is small & 0.6 \\
\hline If hcaw is Vbig then priority is Vsmall & 0.6 \\
\hline If daw is Vshort then priority is Vbig & 0.5 \\
\hline If daw is short then priority is big & 0.5 \\
\hline If daw is med then priority is med & 0.5 \\
\hline If daw is big then priority is small & 0.5 \\
\hline If daw is Vbig then priority is Vsmall & 0.5 \\
\hline
\end{tabular}

Table 2. Simulation condition

\begin{tabular}{|l|c|}
\hline \multicolumn{1}{|c|}{ Parameter } & Description \\
\hline Floor Number & 20 \\
\hline Number of Elevators & 4 \\
\hline Number of persons & 50 Persons per minute \\
\hline Capacity of Elevator & 26 Persons \\
\hline Elevator Speed & 1 floor per second \\
\hline Stopping Time & 4 seconds \\
\hline
\end{tabular}

The simulation conditions, as given in Table 2, are applied on the Simulink model of the EGCS in which the elevator with the highest priority is selected out of all the four elevators.

\section{Result and Discussion}

These results are taken by giving the various calls to the elevator system at a different time interval such as at time interval 0.2 the given call is the 3rd floor and so on interval 1 the given call is $15^{\text {th }}$ floor. Figure 4 shows the input parameters. Figure 5 shows the maximum priority of the elevator with respect to the time interval and their calls. Figure 6 shows the priority of each elevator with respect to the time interval and their calls. 
International Journal of Mathematical, Engineering and Management Sciences

Vol. 4, No. 1, 77-84, 2019

https://dx.doi.org/10.33889/IJMEMS.2019.4.1-007

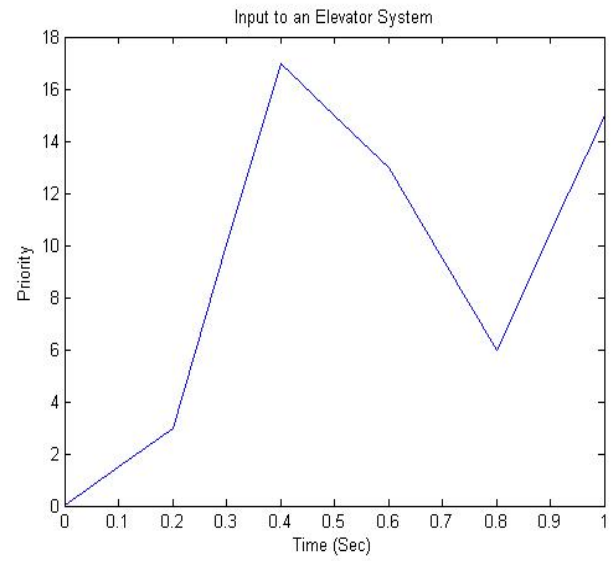

Figure 4. Input parameters

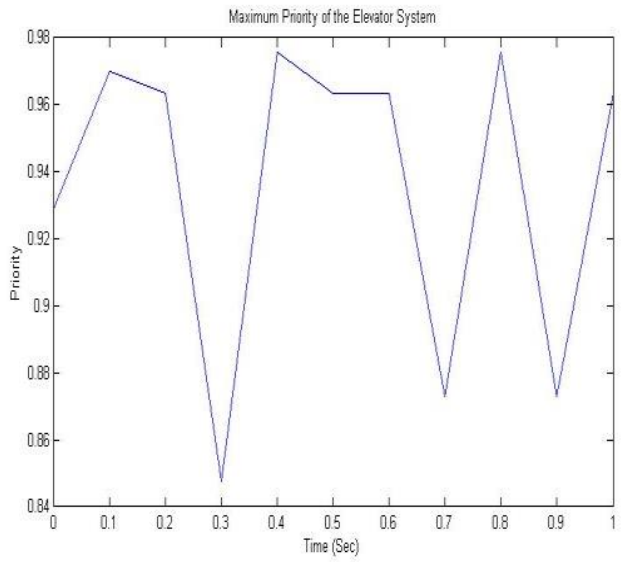

Figure 5. Maximum priority

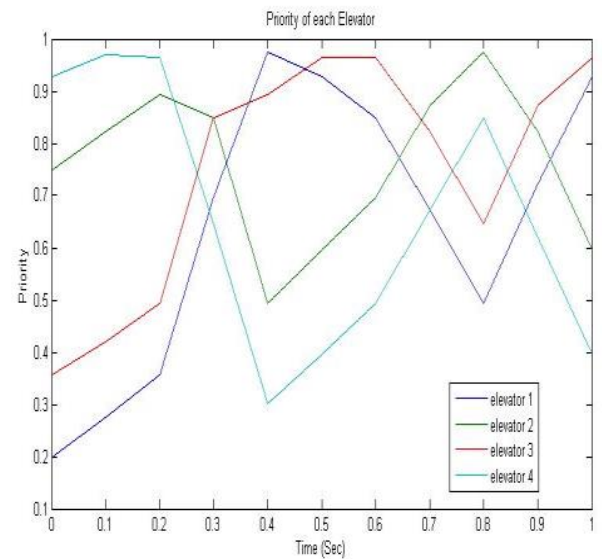

Figure 6. Priority of each elevator 
International Journal of Mathematical, Engineering and Management Sciences

Vol. 4, No. 1, 77-84, 2019

https://dx.doi.org/10.33889/IJMEMS.2019.4.1-007

\section{Conclusion}

In this work, a simulation model is proposed with fuzzy logic control to choose the most appropriate elevator for a hall call generated by passenger/user. The input to the simulation is given by the user and the necessary calculation is done with the fuzzy logic controller. The data obtained from the simulation model and the input source provided to the fuzzy based controller priority of each elevator car can be calculated. The final command is given to the maximum priority of the elevator car as per the hall call operation. This work will help in avoiding any sort of error as it is based on the fuzzy logic which is proven to be a composed controller and used in various industrial applications.

\section{Conflict of Interest}

The authors confirm that this article contents have no conflict of interest.

\section{Acknowledgement}

The authors would like to express their sincere thanks to the referee and for their valuable suggestions towards to the improvement of the paper.

\section{References}

Chaube, S., \& Singh, S. B. (2016a). Fuzzy reliability of two-stage weighted-k-out-of-n systems with common components. International Journal of Mathematical, Engineering and Management Sciences, 1(1), 41-51.

Chaube, S., \& Singh, S. B. (2016b). Fuzzy reliability theory based on membership function. International Journal of Mathematical, Engineering and Management Sciences, 1(1), 34-40.

Deying, G., \& Dongmei, Y. (2010, March). Study on fuzzy algorithm of elevator group control system. In Challenges in Environmental Science and Computer Engineering (CESCE), 2010 International Conference on (Vol. 1, pp. 366-369). IEEE.

Imasaki, N., Kubo, S., Nakai, S., Yoshitsugu, T., Kiji, J. I., \& Endo, T. (1995). Elevator group control system tuned by a fuzzy neural network applied method. In Fuzzy Systems, 1995. International Joint Conference of the Fourth IEEE International Conference on Fuzzy Systems and The Second International Fuzzy Engineering Symposium., Proceedings of 1995 IEEE Int (Vol. 4, pp. 1735-1740). IEEE.

Ishikawa, T., Miyauchi, A., \& Kaneko, M. (2000). Supervisory control for elevator group by using fuzzy expert system which also address travelling time. Proceedings of IEEE International Conference on Industrial Technology 2000 (IEEE Cat. No.00TH8482), 2(1), (pp. 87-94).

Kickert, W. J. M., \& Mamdani, E. H. (1993). Analysis of a fuzzy logic controller. In Readings in Fuzzy Sets for Intelligent Systems (pp. 290-297).

Kim, T. S., Kwon, J. K., \& Sung, D. K. (2000). Mobility modeling and traffic analysis in three-dimensional high-rise building environments. IEEE Transactions on Vehicular Technology, 49(5), 1633-1640.

King, S. S., \& Bouketir, O. (2008). Simulation of a four-car elevator operation using MATLAB. Modern Applied Science, 2(6), 100-109.

Lee, C. C. (1990). Fuzzy logic in control systems: fuzzy logic controller. IEEE Transactions on Systems, Man, and Cybernetics, 20(2), 404-418. 
International Journal of Mathematical, Engineering and Management Sciences

Vol. 4, No. 1, 77-84, 2019

https://dx.doi.org/10.33889/IJMEMS.2019.4.1-007

Nikovski, D., \& Brand, M. (2004). Exact calculation of expected waiting times for group elevator control. IEEE Transactions on Automatic Control, 49(10), 1820-1823.

Patjoshi, R. K., \& Mahapatra, K. K. (2010). FPGA implementation of fuzzy logic controller for elevator group control system. Proceddings of the International Conference on Future Engineering Trends (ICFET 2K10), SSE, Saveetha University, India.

Sugeno, M. (1985). An introductory survey of fuzzy control. Information Sciences, 36(1-2), 59-83.

Tanaka, S., \& Watanabe, M. (2009, August). Optimization-based collision avoidance in multi-car elevator systems. In ICCAS-SICE, 2009 (pp. 764-769). IEEE.

Zadeh, L. A. (1973). Outline of new approach to the analysis complex systems and decision processes. IEEE Transactions on Systems, Man, and Cybernetics, SMC-3(1), 28-44. 